\title{
Wave transmission in time- and space-variant helicoidal phononic crystals
}

\author{
F. Li, ${ }^{1}$ C. Chong, $, 2,3,{ }^{*}$ J. Yang,,${ }^{1} \dagger$ P. G. Kevrekidis, ${ }^{2}$ and C. Daraio ${ }^{3,4}$ \\ ${ }^{1}$ Aeronautics and Astronautics, University of Washington, Seattle, Washington 98195-2400, USA \\ ${ }^{2}$ Department of Mathematics and Statistics, University of Massachusetts, Amherst, Massachusetts 01003-4515, USA \\ ${ }^{3}$ Department of Mechanical and Process Engineering (D-MAVT), Swiss Federal Institute of Technology (ETH), 8092 Zurich, Switzerland \\ ${ }^{4}$ Engineering and Applied Science, California Institute of Technology, Pasadena, CA 91125, USA
}

(Received 26 August 2014; published 4 November 2014)

\begin{abstract}
We present a dynamically tunable mechanism of wave transmission in one-dimensional helicoidal phononic crystals in a shape similar to DNA structures. These helicoidal architectures allow slanted nonlinear contact among cylindrical constituents, and the relative torsional movements can dynamically tune the contact stiffness between neighboring cylinders. This results in cross-talking between in-plane torsional and out-of-plane longitudinal waves. We numerically demonstrate their versatile wave mixing and controllable dispersion behavior in both wavenumber and frequency domains. Based on this principle, a suggestion toward an acoustic configuration bearing parallels to a transistor is further proposed, in which longitudinal waves can be switched on and off through torsional waves.
\end{abstract}

DOI: 10.1103/PhysRevE.90.053201

PACS number(s): 46.40.-f, 62.30.+d, 45.70.-n, 05.45.-a

\section{INTRODUCTION}

Phononic crystals (PCs) are spatially periodic structures that can manipulate acoustic waves more effectively compared to natural materials [1,2]. Intense recent research efforts along this direction have shown that the acoustic characteristics of PCs depend on their given material properties, geometrical configurations, and boundary conditions. For example, the frequency band structures of PCs can be modified by changing their structural compositions or by the application of external fields [3-5]. Although tunable PCs have been investigated both theoretically and experimentally, their acoustic properties are typically fixed by their initial design parameters and are not allowed to vary adaptively, which limits the breadth of their potential applications. Previous studies explored the possibility of altering wave transmission characteristics in situ by using time-varying material properties [6] and by exploiting amplitude-dependent responses of their nonlinear constituents [7-9]. However, dynamically tunable PCs are relatively unexplored, and wave propagation mechanisms in time- and space-variant PCs remain largely unknown.

Recently, the specific paradigm of granular crystals based on the assembly of discrete particles has attracted significant attention due to their nonlinearity stemming from Hertzian contact interaction $[10,11]$. The tunability of this nonlinearity from the weakly to the highly nonlinear regime involves a degree of freedom that is significant in this regard [10-14]. Particularly, PCs with cylindrical elements have shown their dynamic versatility in controlling the speed of nonlinear waves [15] and manipulating the cutoff frequencies of band gaps over remarkably wide ranges [16]. These studies leveraged the variations of contact stiffness among slanted cylinders by changing their alignment angles in a static manner.

In the present work, we report on the dynamic manipulation of wave propagation modes in one-dimensional (1D) PCs made of helically stacked cylinders defined as helicoidal phononic crystals (HPCs) herein. We impose in-plane torsional waves

\footnotetext{
*cchong@ethz.ch

†jkyang@aa.washington.edu
}

to HPCs by systematically perturbing the alignment angles of cylinders in the temporal and spatial domains. This results in dynamic variations of axial contact stiffness in the helicoidal structures, thereby making longitudinal waves coupled with the torsional waves. In principle, this phonon-phonon scattering (i.e., wave mixing) effect is equivalent to optical Brillouin scattering [17]. Such dynamic cross-talking between torsional and longitudinal waves offers an unprecedented controllability over wave transmission in PCs, exhibiting fundamentally distinct characteristics in comparison to conventional PCs with a fixed landscape of wave dispersions. Herein, we demonstrate, for the first time, versatile manipulation of wave dispersion mechanisms of a certain wave mode via another by using time- and space-variant HPCs. In particular, we employ three distinct schemes: one in which the HPCs are space-independent, varying solely with time; one in which they are time-independent but are varying with space; and one in which both the tunability in space and in time are employed concurrently. Finally, although acoustic diodes have been proposed $[18,19]$, we note that an acoustic transistor has not been realized. Based on HPCs, we propose a configuration bearing characteristics of a transistor, in that a longitudinal wave can be controlled actively by a torsional wave.

Our presentation is structured as follows: In Sec. II, we analyze the general model and give details of the availability of parametric variations. In Sec. III, we consider time-dependent, space-independent helicoidal configurations, in Sec. IV timeindependent but spatially dependent ones are studied, and in Sec. V we combine both variations. In Sec. VI, we provide a potential application of the HPCs with a view toward a future implementation of an acoustic transistor. Finally, in Sec. VII, we summarize our findings and provide some directions for future study.

\section{MODEL}

The HPC investigated in this study is arranged in a DNAlike helical architecture with increasing alignment angles (we denote the absolute angle of the $n$th particle with respect to the first particle as $\alpha_{n}$ ). The schematic of the HPC is shown 


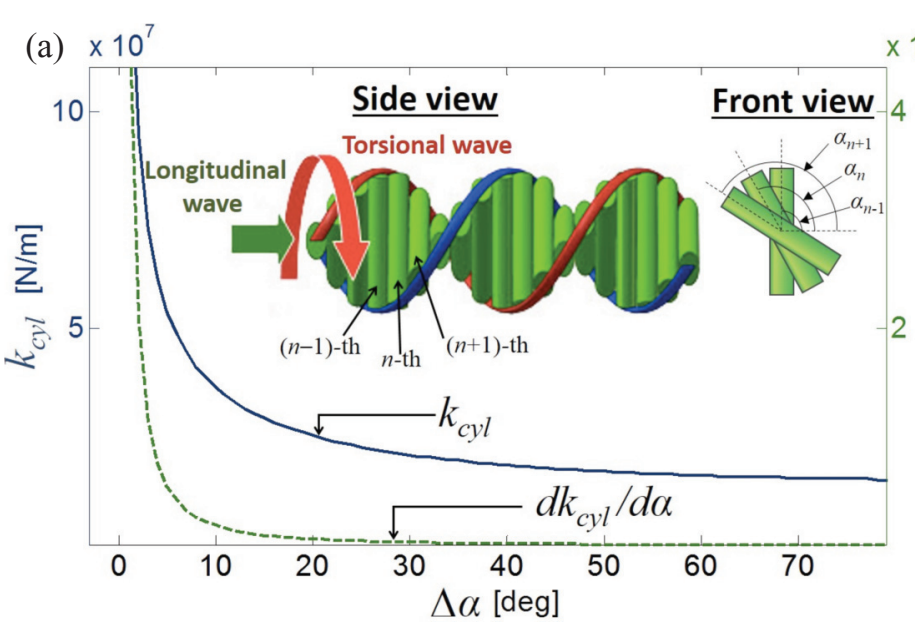

(b)

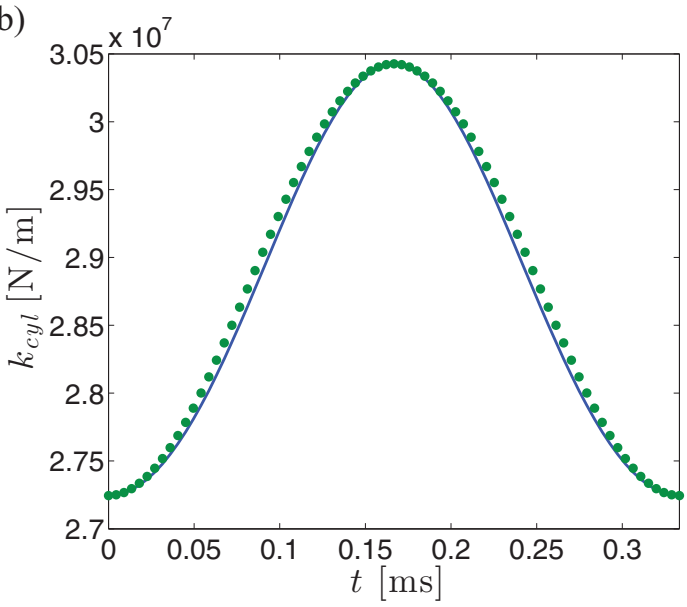

FIG. 1. (Color online) (a) Stiffness and sensitivity of the cylindrical Hertzian contact as a function of alignment angles. A schematic of the HPC composed of a 1D chain of helicoidally stacked cylindrical particles under precompression is shown in the inset. (b) The stiffness coefficient $k_{\text {cyl }}$ (solid blue line) and its single harmonic approximation based on its Taylor expansion (green markers). The parameter values in this case are $\alpha_{0}=10^{\circ}, A_{\alpha}=1^{\circ}$, and $f_{\alpha}=3 \mathrm{kHz}$.

in the inset of Fig. 1(a). Each cylindrical element supports both longitudinal and rotational movements. Longitudinal waves propagate along the helical chain through the point contact among neighboring elements, while torsional waves are imposed on the HPC at will by actuating each one of the cylinders independently. We neglect the torsional elasticity or friction at the contact. The relative angle between neighboring cylinder particles is $\Delta \alpha_{n}=\left|\alpha_{n}-\alpha_{n-1}\right|$. The contact force between two adjacent cylindrical particles is expressed by the Hertzian law $F=k_{\text {cyl }}(\Delta \alpha) \delta^{3 / 2}$ for $\Delta \alpha \neq 0$, where $F$ and $\delta$ are the contact force and displacement, respectively. The contact stiffness coefficient is given as a function of the angle of other relevant material parameters as [20]

$$
\begin{aligned}
k_{\mathrm{cyl}}(\Delta \alpha)= & \frac{\pi \sqrt{2} Y}{3\left(1-v^{2}\right) e K(e)^{3 / 2}}\left\{\left[\left(\frac{1}{1-e^{2}}\right) E(e)-K(e)\right]\right. \\
& \times[K(e)-E(e)]\}^{1 / 4} .
\end{aligned}
$$

Here $K(e)$ and $E(e)$ are the complete elliptic integrals of the first and second kinds, respectively, and $e=$ $\sqrt{1-\{\cos (\Delta \alpha) /[1+\cos (\Delta \alpha)]\}^{4 / 3}}$ is the eccentricity of the elliptical contact area between two cylindrical particles. Furthermore, $Y$ represents the Young's modulus and $v$ the Poisson ratio. The contact stiffness $k_{\text {cyl }}$ is sensitive to the relative alignment angle $\Delta \alpha$ between adjacent cylindrical particles [see Fig. 1(a)], implying that the dynamics of the axial and rotational motions of HPCs are coupled. The longitudinal motion of the HPC can be written in terms of both rotational angle $\Delta \alpha_{n}$ and axial displacement $u_{n}$ of the $n$th element:

$$
\begin{aligned}
M \ddot{u}_{n}= & k_{\mathrm{cyl}}\left(\Delta \alpha_{n}\right)\left[\delta_{n}+u_{n-1}-u_{n}\right]_{+}^{3 / 2} \\
& -k_{\mathrm{cyl}}\left(\Delta \alpha_{n+1}\right)\left[\delta_{n+1}+u_{n}-u_{n+1}\right]_{+}^{3 / 2},
\end{aligned}
$$

where $M$ is the mass of a cylindrical unit and $\delta_{n}$ is the deformation at static equilibrium between the $n$th and $(n+$ 1)th particles resulting from the precompression force $F_{0}=$ $k_{\text {cyl }}\left(\Delta \alpha_{n}\right) \delta_{n}^{3 / 2}$. The bracket is defined by $[x]_{+}=\max (0, x)$, denoting that there are no tensional forces among cylinders. For the numerical results reported in this study, we consider HPCs composed of 200 fused quartz cylinders with diameter $18.0 \mathrm{~mm}$ and height $18.0 \mathrm{~mm}$. The mass, Young's modulus, and Poisson's ratio are $M=10 \mathrm{~g}, Y=72 \mathrm{GPa}$, and $v=0.17$, respectively. The precompression $\left(F_{0}\right)$ of the chain is assumed to be $20 \mathrm{~N}$. The dimensions, material properties, and boundary conditions are based on the parameters used in the earlier experimental study of [16].

For small relative displacements $\left|u_{n-1}-u_{n}\right| \ll \delta_{n}$, the stiffness of the Hertzian contact can be linearized [14]. Thus, we can infer dispersion properties of this system by studying the linearized equations of motion,

$$
\begin{aligned}
M \ddot{u}_{n}= & k_{\operatorname{lin}}\left(\Delta \alpha_{n}\right) u_{n-1}-\left[k_{\operatorname{lin}}\left(\Delta \alpha_{n}\right)+k_{\operatorname{lin}}\left(\Delta \alpha_{n+1}\right)\right] u_{n} \\
& +k_{\operatorname{lin}}\left(\Delta \alpha_{n+1}\right) u_{n+1},
\end{aligned}
$$

where the linear stiffness coefficient is given as $k_{\operatorname{lin}}(\Delta \alpha)=$ $3 / 2 k_{\text {cyl }}(\Delta \alpha) \delta_{n}^{1 / 2}$. While the nonlinear case of Eq. (1) is extremely interesting in its own right, and we will return to it in Sec. VI, for the purposes of the present work, we will restrict most of our considerations to the linearized problem, i.e., Eq. (2).

We first consider as a reference case a regular HPC without introducing any dynamic perturbations of angles (i.e., the standard, homogeneous case). More specifically, cylindrical angles in the helicoidal chain increase linearly by $\alpha_{0}$ (i.e., $\alpha_{n}=n \alpha_{0}$ ), and thus the relative angles between neighboring particles remain constant $\left(\Delta \alpha=\alpha_{0}\right)$. Then, the axial stiffness $k_{\text {cyl }}$ (and hence $k_{\text {lin }}$ ) is the same along the chain, which is equivalent to a standard, homogeneous chain [10]. In this instance, the linear problem, Eq. (2), is solved by means of the Fourier mode plane wave ansatz $u_{n}=\exp (i k n+i \omega t)$, where the wavenumber $k$ and the frequency $\omega$ satisfy the dispersion relation

$$
\omega(k)^{2}=2 k_{\operatorname{lin}}\left(\alpha_{0}\right)[1-\cos (k)] / M,
$$

such that the maximum allowable frequency (i.e., the cutoff frequency) is $f_{\text {cutoff }}=\sqrt{\frac{k_{\text {lin }}}{M}} / \pi$. The computation of dispersion relations in the case of dynamic HPCs is considerably more 
involved. Thus, we now turn, in Secs. III-V, to the reformulation of results on the spectra of spatially and/or temporally periodic linear difference operators and compare these semianalytical results to full numerical simulations of the nonlinear model Eq. (1) in the case of small relative displacements.

\section{TIME-VARIANT, SPACE-INDEPENDENT HPCS}

We now consider a standing torsional wave in the helicoidal chain, where the angles of the cylindrical particles are given by

$$
\alpha_{n}=n \alpha_{0}+n A_{\alpha} \cos \left(\omega_{\alpha} t\right),
$$

where $\omega_{\alpha}=2 \pi f_{\alpha}$ is the frequency of the standing torsion wave. These angular variations result in dynamic stiffness changes in the axial direction. In order to simplify the analysis, we assume $\left|A_{\alpha}\right| \ll\left|\alpha_{0}\right|$, such that we may Taylor expand $k_{\text {cyl }}(\Delta \alpha)$ with respect to $A_{\alpha} \cos \left(\omega_{\alpha} t\right)$, yielding an expression for the linear stiffness that is composed of a single harmonic

$$
k_{\operatorname{lin}} \approx \tilde{\alpha}_{0}+\tilde{A}_{\alpha} \cos \left(\omega_{\alpha} t\right)
$$

where

$$
\tilde{\alpha}_{0}=\frac{3}{2} k_{\mathrm{cyl}}\left(\alpha_{0}\right) \delta_{0}^{1 / 2} \quad \tilde{A}_{\alpha}=\frac{3}{2} k_{\mathrm{cyl}}^{\prime}\left(\alpha_{0}\right) A_{\alpha} \delta_{0}^{1 / 2},
$$

where the prime denotes the derivative with respect to the argument. See, e.g., Fig. 1(b) for the validity of this approximation when $\left|A_{\alpha}\right| \ll\left|\alpha_{0}\right|$. In order to compute the dispersion relationship of Eq. (2) with the time-dependent stiffness coefficient, Eq. (5), we make use of the discrete Fourier transform

$$
\hat{u}(k, t)=\sum_{n \in \mathbb{Z}} u_{n}(t) e^{i k n},
$$

where $\hat{u}(k, t)=\hat{u}(k+\pi, t)$. Thus, Eq. (2) can be rewritten as

$$
\partial_{t}^{2} \hat{u}(k, t)=-\omega(k)^{2}\left[1+\frac{\tilde{A}_{\alpha}}{\tilde{\alpha}_{0}} \cos \left(\omega_{\alpha} t\right)\right] \hat{u}(k, t),
$$

where $\omega(k)$ is the dispersion relation in the homogeneous case; see Eq. (3). For each $k$, Eq. (6) represents the well-studied

(a)

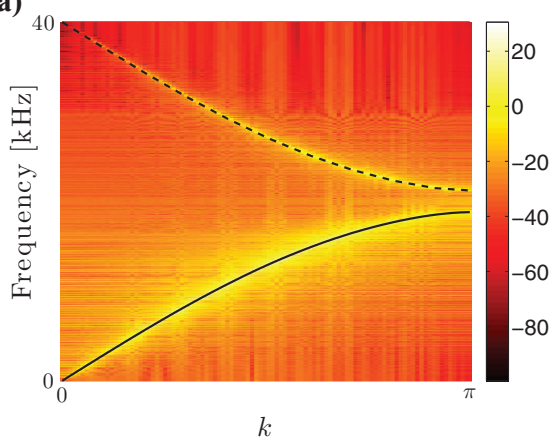

(b)

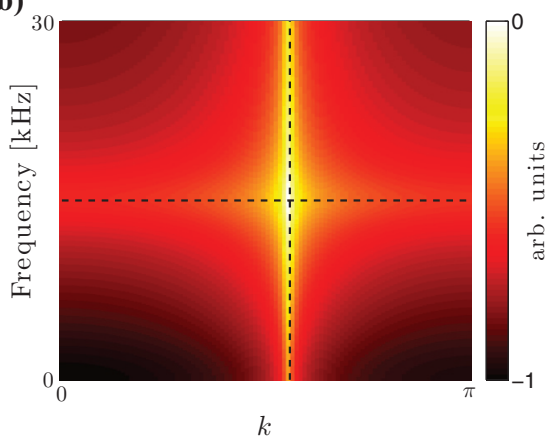

Mathieu equation, whose general solution can be found using Floquet theory [21]:

$$
\hat{u}(k, t)=c_{1} e^{\mu_{1}(k) t} p_{1}(t)+c_{2} e^{\mu_{2}(k) t} p_{2}(t),
$$

where $\mu_{1}(k)$ and $\mu_{2}(k)$ are the Floquet exponents, $c_{1}$ and $c_{2}$ are arbitrary constants, and $p_{1}$ and $p_{2}$ are functions with period $T_{\alpha}=2 \pi / \omega_{\alpha}$. For the Mathieu equation, stability is only possible if the Floquet exponents are purely imaginary. Typically, the imaginary part of the exponent [which we denote $\sigma(k)]$ is incommensurate with the frequency $\omega_{\alpha}$, and thus the dynamics is not periodic. The unstable and stable regions are separated in parameter space by periodic solutions. Thus, the stability boundaries can be determined by finding parameter values where periodic solutions are possible. This can be achieved by substituting the Fourier series representation of a periodic solution into the Mathieu equation and demanding that the Fourier coefficients be nontrivial. Doing so leads to the well-known determinant condition to determine the stability regions of the Mathieu equation [22]. In the $\left[\frac{\omega_{\alpha}}{2 \omega(k)}, \frac{\tilde{A}}{\tilde{\alpha}_{0}}\right]$ plane, the regions of instability are wedge-like and originate (i.e., when $\tilde{A} / \tilde{\alpha}_{0}=0$ ) at the values

$$
\frac{\omega_{\alpha}}{2 \omega(k)}=\frac{1}{j}
$$

where $j \in \mathbb{Z}^{+}$. Since in our setting $|\omega(k)|$ increases monotonically from zero as $k$ increases, we need only to consider the first stability boundary [i.e., the one originating at $\frac{\omega_{\alpha}}{2 \omega(k)}=1$ ]. This boundary can be approximated analytically, leading to the following condition for stability in our setting:

$$
\frac{\omega_{\alpha}}{2 \omega(\pi)}>1+\left|\frac{\tilde{A}}{4 \tilde{\alpha}_{0}}\right| \text {. }
$$

Figures 2(a) and 2(b) show the Fourier transform in the spatial and temporal domains of a solution of Eq. (1) for parameter values satisfying and violating condition Eq. (8), respectively. For the unstable case considered in Fig. 2(b), only the first instability region is entered. Thus, although the solutions grow

FIG. 2. (Color online) (a) Superposition of (i) Floquet exponents $\sigma(k) /(2 \pi)$ (solid line) and a first-order harmonic $\sigma(k) / 2 \pi+f_{\alpha}$ [dashed line, see Eq. (10)] versus the wavenumber $k$ [parameter values are $\alpha_{0}=10, A_{\alpha}=5$, and $f_{\alpha}=40 \mathrm{kHz}$, which satisfy the stability condition Eq. (8)], and (ii) PSD [dB] of velocity component of the solution of Eq. (1) obtained by imposing a chirped pulse (0 to $30 \mathrm{kHz}) \mathrm{with}$ an amplitude of $5 \mathrm{~nm}$ on the left end of a resting chain. (b) PSD [arbitary units] of velocity component of the solution with the unstable parameter values $\alpha_{0}=10, A_{\alpha}=5$, and $f_{\alpha}=30 \mathrm{kHz}$. The vertical dashed line corresponds to the wavenumber yielding the Floquet exponent with the largest real part. The horizontal line is the corresponding imaginary part of that exponent. The chain was excited in the same way as described for panel (a). (c) Same as described for panel (a), but with the parameter values $\alpha_{0}=10, A_{\alpha}=1$, and $f_{\alpha}=3 \mathrm{kHz}$, which do not satisfy the stability condition Eq. (8). However, in this case, the wavenumbers do not fall into regions of instability of the Mathieu equation due to the finite nature of the simulations (see text), and thus the observed dynamics are stable. The chain was excited in the same way as described for panel (a). 
without bound, one can detect spectral concentration within the range of wavenumbers that fall in the instability region. For a chain of finite length $L$, the span of wavenumbers becomes discrete, e.g., $k_{n}=n \pi /(L+1)$ in the case of fixed boundary conditions. Thus, it is possible that each wavenumber avoids each instability region of the Mathieu equation. For example, if we assume fixed boundary conditions and $|\tilde{A}| \ll\left|\tilde{\alpha}_{0}\right|$, then Eq. (7) implies that the quantity

$$
\frac{\omega_{\alpha}}{2 \omega[n \pi /(L+1)]}, \quad|n| \leqslant L
$$

should not be in a neighborhood of $1 / j$ for each $j \in \mathbb{Z}^{+}$in order to achieve stability. See Fig. 2(c) for an example.

In the case that the Mathieu equation is stable, we have $\mu_{1,2}(k)= \pm i \sigma(k)$, where $\sigma(k) \in \mathbb{R}$ and $p_{1}(t)=\bar{p}_{2}(t)$. Since $p_{1}$ has period $T_{\alpha}$, we have the following solution:

$$
\hat{u}(k, t)=\sum_{m \in \mathbb{Z}} a_{m}(k) e^{i\left(\sigma(k)+\omega_{\alpha} m\right) t}, \quad a_{m} \in \mathbb{C},
$$

where the Fourier coefficients $a_{m}(k)$ of the function $p_{1}(t)$ satisfy

$$
\frac{a_{m}(k)}{a_{m-1}(k)+a_{m+1}(k)}=\frac{\tilde{A}_{\alpha}}{2 \tilde{\alpha}_{0}}\left\{\frac{\omega(k)^{2}}{\left[\sigma(k)+\omega_{\alpha} m\right]^{2}-\omega(k)^{2}}\right\} .
$$

Our analysis shows that the longitudinal wave, interacting with the periodic stiffness variations, produces frequency shifts similar to optical Brillouin scattering [17]. This implies that the HPCs can realize wave mixing effects, whereby interactions between longitudinal and torsional waves produce extra dispersion modes in variable frequencies. For example, in Fig. 2(a) the value of $f_{\alpha}$ is such that the curve $-\sigma(k) / 2 \pi$ is shifted into view (i.e. it becomes the next closest dispersion branch). This is in contrast to Fig. 2(c) where $f_{\alpha}$ is such that the principle dispersion branch $\sigma(k) / 2 \pi$, when shifted the amount $f_{\alpha}$, remains in view (i.e. is the next closest dispersion branch). Thus, the newly created modes in time-variant HPCs can effectively up-shift cutoff frequencies ( $f_{\text {cutoff }} \rightarrow f_{\text {cutoff }}+f_{\alpha}$ ). This is especially useful for application purposes, a theme that we will revisit in Sec. VI. It is also relevant to point out that $a_{ \pm 1}$ associated with $\sigma(k) \pm \omega_{\alpha}$ already bear an amplitude nearly two orders of magnitude smaller than the principal mode $a_{0}$. Hence, it is natural to expect that $a_{ \pm 2}$ are considerably harder to identify in the context, e.g., of Figs. 2(a) and 2(c).

As an additional comment, we should note that here we consider angular periodic variations in the cosinusoidal form, in order to capture the essential characteristics of the impact of this variation (in the spirit also of Fourier decomposition of any periodic variation into such modes). Moreover, the evolution of the modes in this case is described by the Mathieu equation [22], which is well-established in the realm of parametric instabilities. Nevertheless, it would be worthwhile to consider other, special forms of periodic variation within the more broad setup of Hill's equations [23] as generalizations of Eq. (6). A notable example that is worthy to explore from the point of view of deriving explicit analytical conditions for the instability manifestation is that of piecewise constant variations of the angle, along the lines of the well-known, exactly solvable Kronig-Penney model of quantum mechanics [24].

\section{SPACE-VARIANT, TIME-INDEPENDENT HPCS}

We now consider modulating the rotational angles of the helicoidal chain in the spatial domain. Under harmonic perturbations, once again, but now in space, the angles of the cylindrical particles become

$$
\alpha_{n}=n \alpha_{0}+A_{\alpha} \cos \left(k_{\alpha} n\right),
$$

where $A_{\alpha}$ and $k_{\alpha}$ are the modulation amplitude and the wavenumber $\left(k_{\alpha}=2 \pi / N\right.$, where $N$ is the spatial period of angular variations). In this case, the linear stiffness coefficient satisfies

$$
k_{\text {lin }}\left(\Delta \alpha_{n}\right)=k_{\text {lin }}\left(\Delta \alpha_{n+N}\right) .
$$

Thus, rather than use the Fourier transform to compute the dispersion relationship (as in the case of spatially homogeneous media), we use the Bloch transform [25]

$$
\check{u}_{j}(k, t)=\sum_{n \in \mathbb{Z}} u_{n N+j}(t) e^{i k n N},
$$

where $j \in\{1,2, \ldots, N\}$ and $\check{u}_{j}(k, t)=\check{u}_{j}(k+2 \pi / N, t)$. Applying the Bloch transform to Eq. (2) yields

$$
\begin{aligned}
M \partial_{t}^{2} \check{u}_{j}(k, t)= & k_{\operatorname{lin}}\left(\Delta \alpha_{j}\right) \check{u}_{j-1}(k, t) \\
& -\left[k_{\operatorname{lin}}\left(\Delta \alpha_{j}\right)+k_{\operatorname{lin}}\left(\Delta \alpha_{j+1}\right)\right] \check{u}_{j}(k, t) \\
& +k_{\operatorname{lin}}\left(\Delta \alpha_{j+1}\right) \check{u}_{j+1}(k, t) .
\end{aligned}
$$

This system of $N$ equations has solutions of the form $\check{u}_{j}(k, t)=\exp \left[i j k+i \omega_{B}(k) t\right] f_{j}(k)$, where $\mathbf{f}$ and $\omega_{B}$ satisfy the eigenvalue problem

$$
-\omega_{B}^{2}(k) \mathbf{f}(k)=M^{-1} B(k) \mathbf{f}(k),
$$

where $\mathbf{f}=\left(f_{1}, f_{2}, \ldots, f_{N}\right)^{T}$, and

$$
B(k)=\left(\begin{array}{ccccccc}
b_{1,1} & b_{1,2} & 0 & \ldots & 0 & 0 & b_{1, N} \\
b_{2,1} & b_{2,2} & b_{2,3} & 0 & \ldots & 0 & 0 \\
0 & b_{3,2} & b_{3,3} & b_{3,4} & 0 & \ldots & 0 \\
& & & \ddots & \ddots & & \vdots \\
\vdots & & \ddots & \ddots & & & \\
0 & \ldots & 0 & b_{N-2, N-3} & b_{N-2, N-2} & b_{N-2, N-1} & 0 \\
0 & 0 & \ldots & 0 & b_{N-1, N-2} & b_{N-1, N-1} & b_{N-1, N} \\
b_{N, 1} & 0 & 0 & \ldots & 0 & b_{N, N-1} & b_{N, N}
\end{array}\right),
$$



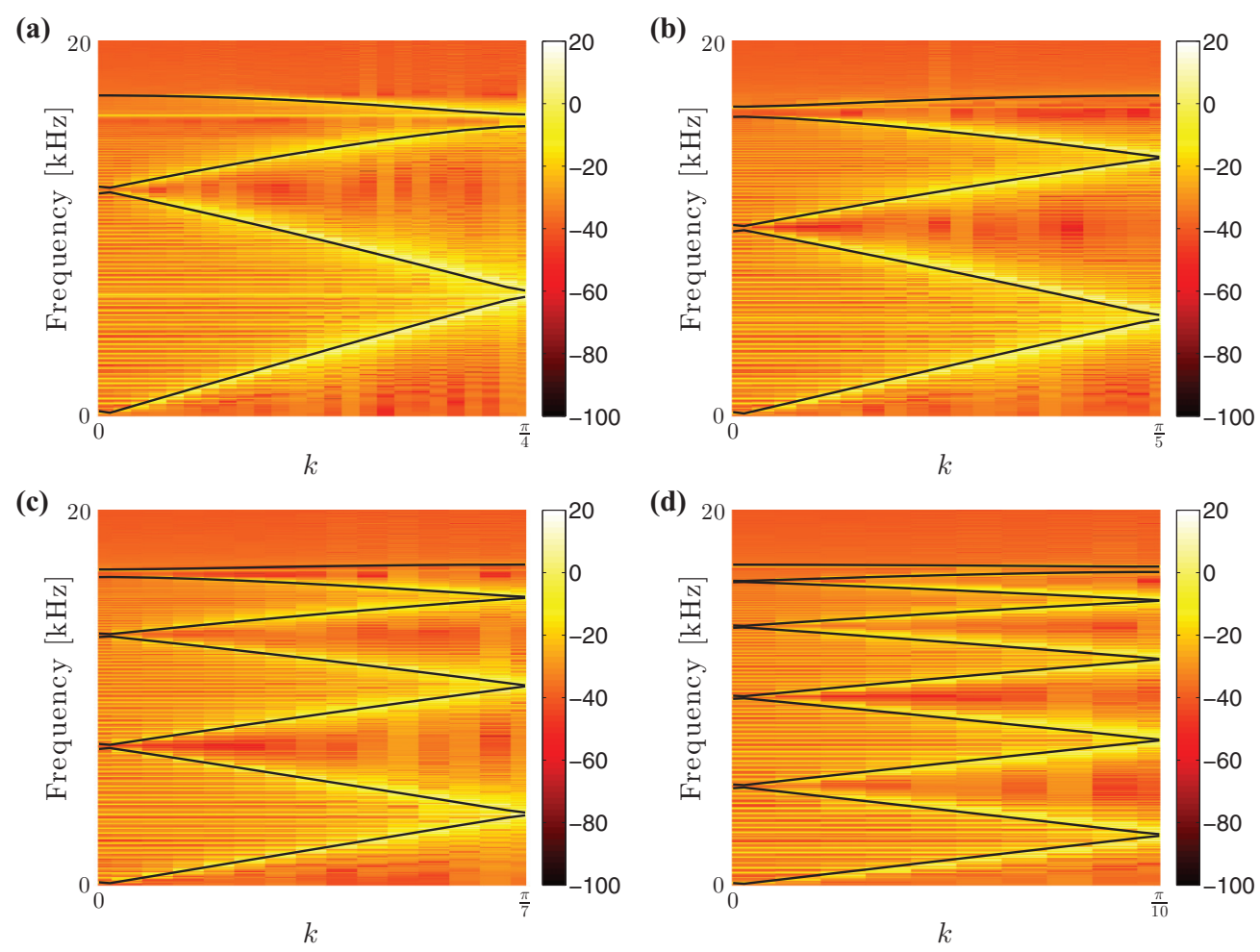

FIG. 3. (Color online) (a) Dispersion relation in the case of space-dependent torsional waves. Shown is the superposition of (i) $N=3$ dispersion curves (solid lines) versus the wavenumber $k \in[0, \pi / N]$ (parameter values are $\alpha_{0}=10^{\circ}$ and $A_{\alpha}=1^{\circ}$ ) and (ii) PSD [dB] of velocity component of the solution of Eq. (1) obtained by imposing a chirped pulse ( 0 to $30 \mathrm{kHz}$ ) with an amplitude of $5 \mathrm{~nm}$ on the left end of a resting chain. (b)-(d) Same as described for panel (a) but with (b) $N=5$, (c) $N=7$, and (d) $N=10$.

with

$$
\begin{aligned}
b_{j, j+1} & =k_{\operatorname{lin}}\left(\Delta \alpha_{j+1}\right) e^{i k}, \\
b_{j, j} & =-\left[k_{\operatorname{lin}}\left(\Delta \alpha_{j}\right)+k_{\operatorname{lin}}\left(\Delta \alpha_{j+1}\right)\right], \\
b_{j, j-1} & =k_{\operatorname{lin}}\left(\Delta \alpha_{j}\right) e^{-i k}, \\
b_{1, N} & =k_{\operatorname{lin}}\left(\Delta \alpha_{1}\right) e^{-i k}, \\
b_{N, 1} & =k_{\operatorname{lin}}\left(\Delta \alpha_{1}\right) e^{i k},
\end{aligned}
$$

for $N>2$, and

$$
\begin{aligned}
& b_{1,2}=k_{\operatorname{lin}}\left(\Delta \alpha_{2}\right) e^{i k}+k_{\operatorname{lin}}\left(\Delta \alpha_{1}\right) e^{-i k} \\
& b_{j, j}=-\left[k_{\operatorname{lin}}\left(\Delta \alpha_{j}\right)+k_{\operatorname{lin}}\left(\Delta \alpha_{j+1}\right)\right] \\
& b_{2,1}=k_{\operatorname{lin}}\left(\Delta \alpha_{1}\right) e^{i k}+k_{\operatorname{lin}}\left(\Delta \alpha_{2}\right) e^{-i k}
\end{aligned}
$$

for $N=2$. If the system parameters are chosen such that $k_{\text {lin }}\left(\Delta \alpha_{j}\right)>0$, then stability follows trivially, since $B$ has real, nonpositive eigenvalues (which can be shown using the Gershgorin circle theorem [26] or by noting that $B$ is a Jacobi operator [27]). Under this assumption, our dispersion relation will be composed of $2 N$ curves ( $N$ of which are nonnegative); see, e.g., Fig. 3(a). We see that multiple wave modes are generated due to the effect of periodic variations of spatial angles and that band gaps appear among newly generated dispersion curves. It should be noted that in other areas of physics, such as, e.g., nonlinear optics [28] and atomic Bose-Einstein condensates [29] (see also references therein), the use of such so-called superlattice potentials is fairly widespread, leading to the formation of minigaps (i.e., gaps within the fundamental band existence in the absence of additional periodicities). Within these minigaps, nonlinear stationary states are also typically sought in these problems.

The space-time evolution of the longitudinal waves can be obtained from the direct numerical integration of Eq. (1). Again, we impose a chirped pulse $(0 \mathrm{~Hz}$ to $30 \mathrm{kHz})$ on one end of the chain with amplitude of $5 \mathrm{~nm}$. The space-time evolution of the HPC's dispersion behavior is calculated via the Fourier transform in the temporal domain and the Bloch transform Eq. (12) in the spatial domain of the bead's velocities; see Fig. 3. While these minigaps become possible for space-dependent torsional waves, we note they become smaller as $N$ increases; see Figs. 3(b)-3(d). As the Brillouin zone shrinks to accommodate the larger periodicity index $N$ (hence the progressively narrower zone ending at $\pi / N$ ), $N$ segments of the dispersion relation "fold" inside this narrower zone with the progressively also narrower minigaps separating them as $N$ increases. This trend is clearly illustrated in Fig. 3.

\section{SPACE- AND TIME-VARYING HPCS}

Here we combine both space- and time-variant effects in the form of traveling torsional waves:

$$
\alpha_{n}=n \alpha_{0}+A_{\alpha} \cos \left(k_{\alpha} n+\omega_{\alpha} t\right)
$$

where $A_{\alpha}$ is the modulation amplitude, $k_{\alpha}$ is the wavenumber ( $k_{\alpha}=2 \pi / N$, where $N$ is the spatial period of angular variations), and $\omega_{\alpha}=2 \pi f_{\alpha}$ is the frequency of the traveling 
(a)

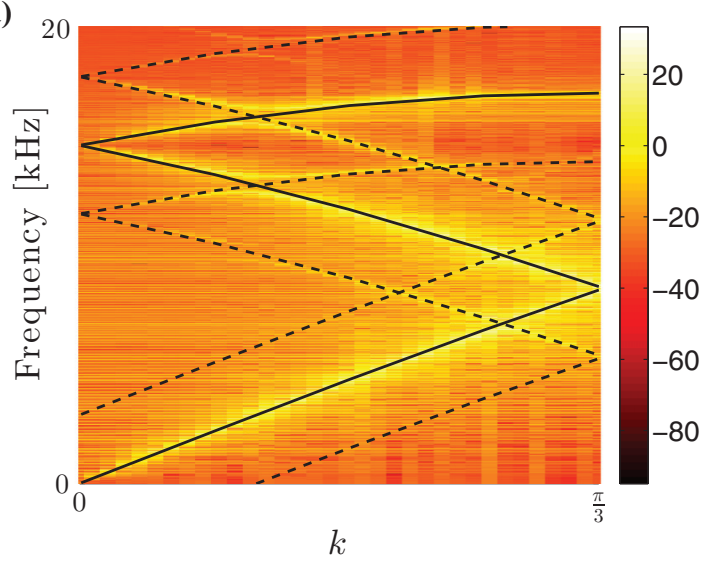

(b)

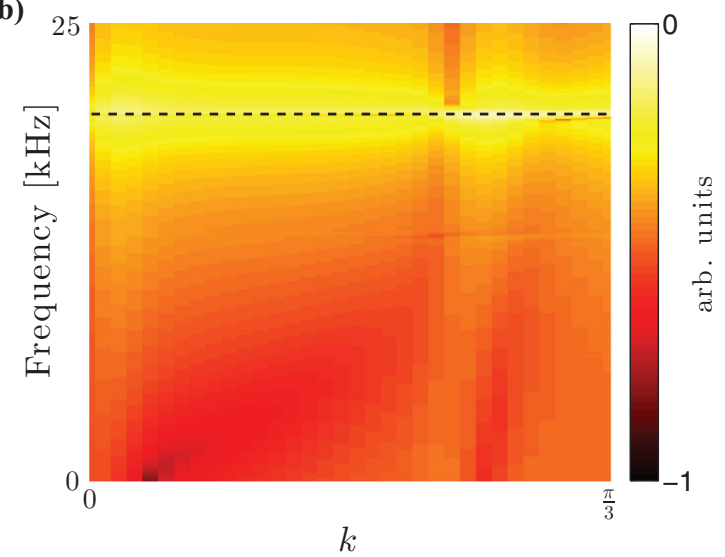

FIG. 4. (Color online) (a) Dispersion relation in the case of space- and time-dependent torsional waves for $\alpha_{0}=10^{\circ}, A_{\alpha}=2^{\circ}, N=3$ and $f_{\alpha}=3 \mathrm{kHz}$. Shown is the superposition of (i) $N=3$ Floquet exponents (solid lines) and the first order harmonic shifts $\sigma(k) / 2 \pi \pm f_{\alpha}$ (dashed lines), and (ii) PSD [dB] of velocity component of the solution of Eq. (1) obtained by imposing a chirped pulse (0 to $30 \mathrm{kHz})$ with an amplitude of $5 \mathrm{~nm}$ on the left end of a resting chain. (b) PSD [arbitrary units] of velocity component of the solution with unstable parameter values $\alpha_{0}=10, A_{\alpha}=5, N=3$ and $f_{\alpha}=40 \mathrm{kHz}$ (note this parameter set is stable for $N=1$, see e.g. Fig. 2(a)). In this case, the Floquet multiplier with the largest modulus occurs for $k \approx 0$. The horizontal line is the imaginary part of the corresponding Floquet exponent. The chain was excited in the same way as in panel (a).

torsion wave. Thus, the linear stiffness coefficient now satisfies

$$
\begin{aligned}
& k_{\operatorname{lin}}\left(\Delta \alpha_{n}\right)(t)=k_{\operatorname{lin}}\left(\Delta \alpha_{n+N}\right)(t), \\
& k_{\operatorname{lin}}\left(\Delta \alpha_{n}\right)(t)=k_{\operatorname{lin}}\left(\Delta \alpha_{n}\right)\left(t+2 \pi / \omega_{\alpha}\right) .
\end{aligned}
$$

Applying the Bloch transform Eq. (12) to Eq. (2) in this case leads to the system of $N$ second-order ODEs with time-periodic coefficients

$$
M \partial_{t}^{2} \check{\mathbf{u}}(k, t)=B(k, t) \check{\mathbf{u}}(k, t),
$$

where $\check{\mathbf{u}}=\left(\check{u}_{1}, \check{u}_{2}, \ldots, \check{u}_{N}\right)^{T}$ and $B$ is defined by Eq. (15) [note in this case $B(k, t)=B\left(k, t+2 \pi / \omega_{\alpha}\right)$ ]. Unlike the timevarying setup considered in Sec. III, there are no analytically tractable conditions for parametric stability of Eq. (16). Thus, one needs to numerically compute the $2 N$ Floquet multipliers to verify that none has modulus greater than unity [where the multiplier is defined as $\left.\exp \left(\mu T_{\alpha}\right)\right]$. Assuming stability, the dispersion curves will have the form $\sigma_{j}(k)+m \omega_{\alpha}$, where $\sigma_{j}(k)$ is the imaginary part of the $j$ th Floquet exponent and $m \in \mathbb{Z}$. Motivated by Fig. 2(b), we choose parameter values with $\left|A_{\alpha}\right| \ll\left|\alpha_{0}\right|$, which leads to a stable system; see, e.g., Fig. 4(a). In this case, as expected, the spectrum is altered by applying an upshift to the cutoff (due to the temporal variance, like in Sec. III) and by increasing the number of primary ( $m=0$ th order) branches (due to the spatial variance, like in Sec. IV). Essentially, in this case, we see a combination of the phenomenologies of Secs. III and IV. The latter is responsible for the formation of the minigaps and the folding of the dispersion relation within the narrower Brillouin zone, while the former is responsible for the emergence of, chiefly, the shifted frequencies by $\pm m \omega_{\alpha}$ (once again, we chiefly observe the ones with $m=1$ ). An interesting observation in this case is the apparent avoided crossing of the Floquet exponents of one branch, with the higher-order harmonics of another branch, as seen in Fig. 4(a). This is a wellknown feature of (typically self-adjoint) matrices representing physical systems under monoparametric variations [30]. It has to do with the fact that degenerate matrices with multiple eigenvalues form a surface of codimension 2 and hence such a crossing cannot be typically created by a monoparametric tuning, such as the one considered, e.g., in Fig. 4(a).

It is also worth noting that the presence of spatial variance can destabilize the system. For example, in Fig. 4(b) we consider the same stable parameter values as in Fig. 2(a), but with a spatial period of $N=3$, leading to instability.

\section{AN APPLICATION BASED ON HPCS: TOWARD AN ACOUSTIC TRANSISTOR}

The realm of HPCs through its tunability can offer a platform that may facilitate the realization of devices analogous to well-established electronic ones. Here, we mention an example of this type in the form of an acoustic transistor. The HPCs can lead to features similar to those of the transistor based on the finding that the dispersive bands of axial waves can be shifted actively by torsional waves. To test this, we numerically excite the first particle in the chain with a Gaussian pulse (bandwidth $b_{w}$ ) and generate longitudinal waves, whose central frequency $f_{L}$ is chosen to be above the cutoff frequency $f_{\text {cutoff }}$. See Fig. 5(a), for example, with the parameter values $f_{L}=20$ $\mathrm{kHz}, b_{w}=1 \mathrm{kHz}, f_{\text {cutoff }}=17.0 \mathrm{kHz}, A_{\alpha}=1^{\circ}, f_{\alpha}=4 \mathrm{kHz}$, and $N=10$. The PSD of the input is the blue curve and the transmitted wave measured at the end of the HPC is the red curve. We find that the longitudinal wave above the cutoff frequency is blocked by the chain in an evanescent manner, and the transmission gain is about $-220 \mathrm{~dB}$. This corresponds to an off state. Now we apply a torsional standing wave and we observe that the amplitude of the longitudinal wave increases by $60 \mathrm{~dB}$ [blue curve in Fig. 5(a)]. This is the on state, which confirms the efficiency of HPCs toward tuning longitudinal propagation, by means of the applied torsional standing wave. Additionally, the transmitted signals contain harmonics of $f_{L}$ and $f_{\alpha}$ such as $f_{L}-f_{\alpha}(16.0 \mathrm{kHz})$ and $f_{L}+f_{\alpha}(24.0 \mathrm{kHz})$. It is important to note that while 

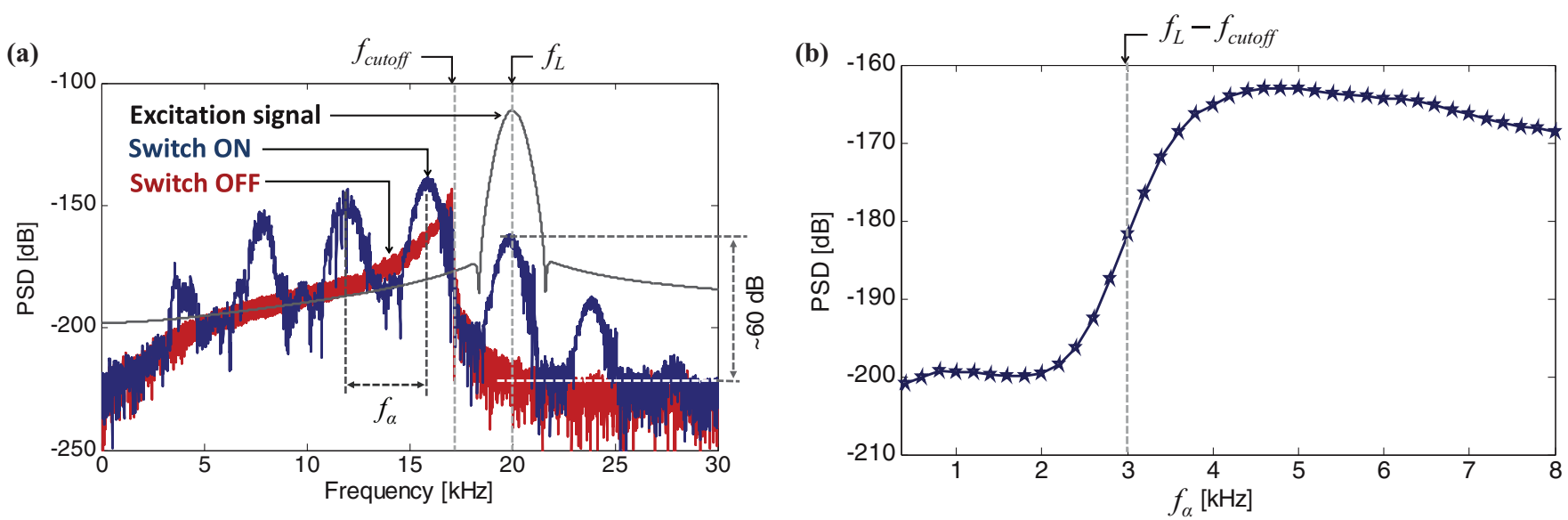

FIG. 5. (Color online) (a) The transistor effect of HPCs. The black curve denotes the Gaussian input signal, while the red (blue) curve corresponds to transmitted signals under the switch off (on) state. $f_{L}$ and $f_{\text {cutoff }}$ are the input and cutoff frequencies. (b) Variation of the transmission of the acoustic wave at frequency $f_{L}$ as a function of the gate frequency $f_{\alpha}$.

this type of signal control is strongly reminiscent of the functionality of a(n acoustic) transistor, nevertheless, our setup does not possess the amplification characteristics encountered in a regular transistor and hence our "device" should not be considered an acoustic transistor per se.

From the working principle of the above setup, the following condition should be satisfied for the transmission of longitudinal waves:

$$
f_{\alpha}>f_{L}-f_{\text {cutoff }} .
$$

Thus, there should exist a lower frequency threshold for a given $f_{L}$ and $f_{\text {cutoff }}$. In order to show this threshold effect, we investigate the frequency dependence of the transmission. We excite a Gaussian pulse with the center frequency $f_{L}=$ $20.0 \mathrm{kHz}$ and bandwidth $b_{w}=120 \mathrm{~Hz}$. The numerical results of the switch transmission with respect to $f_{\alpha}$ are shown in Fig. 5(b). The lower frequency threshold, $f_{L}-f_{\text {cutoff }}$, according to Eq. (17), is plotted by the vertical line. We can see that the transmission of the longitudinal wave increases significantly at $f_{L}-f_{\text {cutoff }}$. This feature is also reminiscent of an electrical transistor: when the gate source voltage is higher than threshold voltage, the conducting channel begins to connect the source and drain of the transistor, allowing a large current to flow.

\section{CONCLUSIONS AND FUTURE CHALLENGES}

We investigated the characteristics of helicoidal phononic crystals (HPCs) in a shape similar to DNA architectures. Based on the Hertzian contact among slanted cylindrical elements, the HPCs develop strong cross-talking between in-plane torsional waves and out-of-plane longitudinal waves. Our (semi)analytical dispersion computations demonstrated that the HPCs exhibit versatile, controllable behavior of longitudinal wave transmission as a function of spatial and temporal variations of torsional waves, which was confirmed against full numerical simulations of the pertinent model. Specifically, it was shown that time-variant HPCs show (symmetric up and down) shifts of dispersive wave modes. This was used to demonstrate that the longitudinal waves can be switched on and off by torsional waves, in an effect reminiscent of the electrical transistor. On the other hand, the space-dependent variant was found to appropriately modify the dispersion relation introducing a reduced Brillouin zone and corresponding minigap within the linear spectrum. The combination of the two effects provided a combination of their influences, as well as additional intriguing features, such as the observed avoided level crossings. Our conclusion is that the time- and space-dependent phononic crystal provides an ideal setting to manipulate acoustic waves by leveraging wave mixing and switching effects and opens the doors for a host of additional studies.

Among the themes of immediate interest, we include the effects of coupling the dynamics of the torsional and longitudinal waves, and considering higher amplitude excitations to test what benefits and disadvantages the nonlinearity of the system introduces. In the latter setting it would be interesting to explore the influence of periodicity in the context of traveling waves $[10,11]$, and the formation of more complex localized breather [31] excitations. It should be noted here that these settings are not only amenable to direct theoretical and numerical investigations, but additionally we believe should be within the immediate grasp of current, state-of-the-art experiments in the field.

\section{ACKNOWLEDGMENTS}

The authors are grateful for the support from the USAFOSR (Grant No. FA9550-12-1-0332), ONR (Grant No. N000141410388), NSF (Grants No. CMMI-1414748, No. CMMI-1000337, No. CMMI-844540), and the ETH Zurich Foundation through the Seed Project ESC-A 06-14. We are grateful to G. Gantzounis for his valuable input on this work.

\section{APPENDIX: DECAY OF FOURIER MODES IN TIME-VARIANT, SPACE-INDEPENDENT HPCS}

The presence of the first-order harmonic shifts [see, e.g., Fig. 2(b)] is what ultimately allows a signal with frequency content lying outside the passband to be transmitted through 
(a)

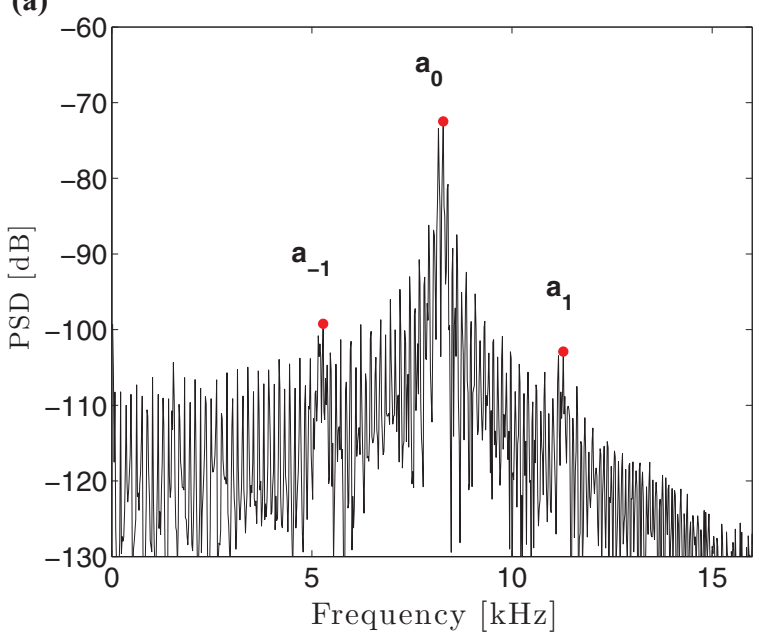

(b)

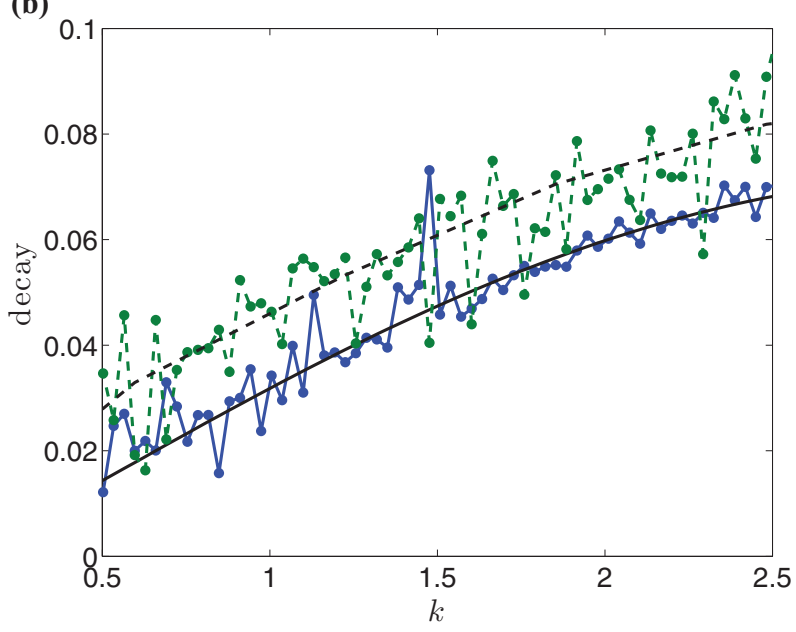

FIG. 6. (Color online) (a) PSD of the displacements $u_{n}$ of the solution shown in Fig. 2(b) for the wavenumber $k=1$. The spectral peaks corresponding to $a_{-1}, a_{0}$ and $a_{1}$ are shown as red points. (b) The decay $a_{1} / a_{0}$ (green dashed line with markers) and $a_{-1} / a_{0}$ (blue line with markers) versus the wavenumber $k$. The analytical predictions from Eq. (18) (black dashed line) and Eq. (19) (solid black line) are also shown.

the chain. Thus, to improve the efficiency of this "transistor effect," it would be desirable to have control over the amplitude of the first-order harmonics relative to the amplitude of the original dispersion curve. This can be achieved by assuming $\left|a_{m}\right| \ll 1$ for $|m|>1$ in Eq. (11), yielding the following prediction:

$$
\begin{gathered}
\frac{a_{1}(k)}{a_{0}(k)}=\frac{\tilde{A}_{\alpha}}{2 \tilde{\alpha}_{0}}\left\{\frac{\omega(k)^{2}}{\left[\sigma(k)+\omega_{\alpha}\right]^{2}-\omega(k)^{2}}\right\}, \\
\frac{a_{-1}(k)}{a_{0}(k)}=\frac{\tilde{A}_{\alpha}}{2 \tilde{\alpha}_{0}}\left\{\frac{\omega(k)^{2}}{\left[\sigma(k)-\omega_{\alpha}\right]^{2}-\omega(k)^{2}}\right\} .
\end{gathered}
$$

To verify this prediction, we numerically solve Eq. (1) and compute the Fourier transform in the time domain for each wavenumber $k$. The largest peak corresponds to the $a_{0}$ mode, which is concentrated around the frequency $\sigma(k)$ and the modes $a_{1}$ and $a_{-1}$ are concentrated at $\sigma(k) \pm \omega_{\alpha}$; see Fig. 6(a), for example. In Fig. 6(b) the decay prediction is shown against numerically computed values for various wavenumbers $k$, where the trend is captured well. Discrepancies stem from the finite nature of the domains considered and the approximation $\left|a_{m}\right|=0$ for $|m|>1$. From Eqs. (18) and (19), we see that if one desires larger spectral peaks at the $m= \pm 1$ harmonics, larger values of $\left|\tilde{A}_{\alpha} / \tilde{\alpha}_{0}\right|$ must be taken. However, this must be weighed against the stability condition Eq. (8), where large values of $\left|\tilde{A}_{\alpha} / \tilde{\alpha}_{0}\right|$ can cause instabilities.
[1] J. V. Sánchez-Pérez, D. Caballero, R. Mártinez-Sala, C. Rubio, J. Sánchez-Dehesa, F. Meseguer, J. Llinares, and F. Gálvez, Phys. Rev. Lett. 80, 5325 (1998).

[2] Z. Y. Liu, Xixiang Zhang, Yiwei Mao, Y. Y. Zhu, Zhiyu Yang, C. T. Chan, Ping Sheng, Science 289, 1734 (2000).

[3] A. Khelif, P. A. Deymier, B. Djafari-Rouhani, J. O. Vasseur, and L. Dobrzynski, J. Appl. Phys. 94, 1308 (2003).

[4] Sz-Chin Steven Lin and T. J. Huang, Phys. Rev. B 83, 174303 (2011).

[5] Jun Liu, Yihui Wu, Feng Li, Ping Zhang, Yongshun Liu, and Junfeng Wu, Europhys. Lett. 98, 36001 (2012).

[6] D. W. Wright and R. S. C. Cobbold, Smart Materials Struct. 18, 015008 (2009).

[7] R. K. Narisetti, M. Ruzzene, and M. J. Leamy, Wave Motion 49, 394 (2012).

[8] J. Yang, S. Dunatunga, and C. Daraio, Acta Mechanica 223, 549 (2012).

[9] P. Wang, F. Casadei, S. Shan, J. C. Weaver, and K. Bertoldi, Phys. Rev. Lett. 113, 014301 (2014).

[10] V. F. Nesterenko, Dynamics of Heterogeneous Materials (Springer-Verlag, New York, 2001).
[11] Surajit Sen, Jongbae Hong, Jonghun Bang, Edgar Avalos, and Robert Doney, Phys. Rep. 462, 21 (2008).

[12] P. G. Kevrekidis, IMA J. Appl. Math. 76, 389 (2011).

[13] G. Theocharis, N. Boechler, and C. Daraio, in Phononic Crystals and Metamaterials, Ch. 6 (Springer Verlag, New York, 2013).

[14] E. B. Herbold, J. Kim, V. F. Nesterenko, S. Wang, and C. Daraio, Acta Mech. 205, 85 (2009).

[15] D. Khatri, D. Ngo, and C. Daraio, Granular Matter 14, 63 (2012).

[16] F. Li, D. Ngo, J. Yang, and C. Daraio, Appl. Phys. Lett. 101, 171903 (2012).

[17] L. Brillouin, Ann. Phys. 17, 88 (1922).

[18] B. Liang, X. S. Guo, J. Tu, D. Zhang, and J. C. Cheng, Nature Mater. 9, 989 (2010).

[19] N. Boechler, G. Theocharis, and C. Daraio, Nature Mater. 10, 665 (2011).

[20] K. L. Johnson, Contact Mechanics (Cambridge University Press, Cambridge, 1985).

[21] Gertrude Blanch, Handbook of Mathematical Functions with Formulas, Graphs, and Mathematical Tables (Dover, New York, 1972). 
[22] Wei-Chau Xie, Dynamic Stability of Structures (Cambridge University Press, Cambridge, 2006).

[23] W. Magnus and S. Winkler, Hill's Equation (Dover Publications, New York, 2004).

[24] I. I. Gold'man and V. D. Krivchenkov, Problems in Quantum Mechanics (Dover Publications, New York, 1993).

[25] M. I. Hussein, Proc. R. Soc. A 465, 2825 (2009).

[26] K. Atkinson, An Introduction to Numerical Analysis (Wiley and Sons, New York, 1989).

[27] G. Teschl, Jacobi Operators and Completely Integrable Nonlinear Lattices (American Mathematical Society, Providence, 2000).
[28] G. Gligorić, A. Maluckov, L. Hadzievski, and B. A. Malomed, Chaos 24, 023124 (2014).

[29] P. J. Y. Louis, E. A. Ostrovskaya, Yu.S. Kivshar, Phys. Rev. A 71, 023612 (2005).

[30] P. D. Lax, Linear Algebra (J. Wiley \& Sons, Hoboken, 2007).

[31] N. Boechler, G. Theocharis, S. Job, P. G. Kevrekidis, Mason A. Porter, and C. Daraio, Phys. Rev. Lett. 104, 244302 (2010); G. Theocharis, N. Boechler, P. G. Kevrekidis, S. Job, Mason A. Porter, and C. Daraio, Phys. Rev. E 82, 056604 (2010); C. Chong, P. G. Kevrekidis, G. Theocharis, and Chiara Daraio ibid. 87, 042202 (2013); C. Chong, F. Li, J. Yang, M. O. Williams, I. G. Kevrekidis, P. G. Kevrekidis, and C. Daraio, ibid. 89, 032924 (2014). 